\title{
Reconfigurable Paramagnetic Microswimmers: Brownian Motion Affects Non-reciprocal Actuation
}

\author{
Di Du, Elaa Hilou and Sibani Lisa Biswal* \\ Department of Chemical and Biomolecular Engineering \\ 6100 Main St. MS 362; Rice University, Houston, TX USA 77005
}

*Corresponding Author:

Sibani Lisa Biswal

6100 Main St. MS 362

Houston, TX 77005

$713-348-6055$

$\underline{\text { biswal@,rice.edu }}$ 


\begin{abstract}
Swimming at low Reynolds number is typically dominated by a large viscous drag, therefore microscale swimmers require non-reciprocal body deformation to generate locomotion. Purcell described a simple mechanical swimmer at the microscale consisting of three rigid components connected together with two hinges. Here we present a simple microswimmer consisting of two rigid paramagnetic particles with different sizes. When placed in an eccentric magnetic field, this simple microswimmer exhibits non-reciprocal body motion and its swimming locomotion can be directed in a controllable manner. Additional components can be added to create a multibody microswimmer, whereby the particles act cooperatively and translate in a given direction. For some multibody swimmers, the stochastic thermal forces fragment the arm, which therefore modifies the swimming strokes and changes the locomotive speed. This work offers insight into directing the motion of active systems with novel time-varying magnetic fields. It also reveals that Brownian motion not only affects the locomotion of reciprocal swimmers that are subject to the Scallop theorem, but also affects that of non-reciprocal swimmers.
\end{abstract}




\section{Introduction}

Reconfigurable materials that can assemble to assume a desired function has been critical towards the development of microscale systems that have the promise for enabling novel self-organizing systems. It is well known that locomotion at the microscale is dominated by interfacial forces, such as friction, drag, and viscous forces ${ }^{1,2}$. The challenge is that microswimmers typically operate in the low Reynolds number $(R e)$ regime, where there is a lack of inertial forces, which are typically exploited for gliding and swimming at the macroscale ${ }^{3,4}$. Nature has evolved microorganisms to swim at low Re by creating deformable bodies that break time-reversibility, such as rotating or beating a tail ${ }^{5,6}$ or waving an elastic arm ${ }^{7}$. For these low Re swimmers, Purcell's "scallop theorem" states that reciprocal motion, such as periodic back and forth flapping motion, cannot lead to net displacement in Newtonian fluids ${ }^{2,8}$. Furthermore, at length scales below tens of microns, effects such as Brownian motion can oftentimes lead to stochastic and unpredictable motion ${ }^{9}$.

Advancements in microfabrication have enabled the engineering of biomimetic microswimmers, such as paramagnetic flagella driven by external fields ${ }^{1,10}$ or biohybrid flagella 11. These artificial swimmers are typically designed after the classical mechanical swimmer, whereby rigid components are linked together by hinges. The simplest mechanical swimmer requires two hinges to translate in a homogeneous Newtonian fluid ${ }^{2}$, where the two hinges link three rigid rods to generate two degrees of freedom, resulting in net propulsion in two-dimension (2-D) if the angles of the two hinges vary in a time-irreversible manner ${ }^{2}$. It has been shown that the hinges can degenerate into stretchable rods in one-dimension (1-D); two stretchable rods connecting three beads can translate when the rods undergo a controlled time-irreversible stretching and contracting motion ${ }^{12-14}$. More recently, microswimmers have been designed to take advantage of the inhomogeneous fluid properties, such as viscosity or strain rate ${ }^{8,15,16}$.

Magnetic actuation has become a prominent method for remote microswimmer power and control due to the ease of applying magnetic forces and torques to magnetizable bodies ${ }^{1,16-19}$. Here we describe an artificial mechanical swimmer at the microscale which consists of two paramagnetic spheres of different sizes that are effectively linked together using a time varying eccentric magnetic field. This magnetic interaction effectively acts as a "hinge" and "stretchable rod" (Fig. 1A) forcing the particles together without the presence of a physical linkage. In this 
untethered microswimmer, the smaller particle makes a well-defined orbit near one side of the larger particle, resulting in the two bodies translating together in a specified direction and velocity that is dependent on the orbit of the smaller particle. This non-reciprocal motion is analogous to a breaststroke, where the arm sweeps near one side of torso without breaking fluid surface, resulting in a stroke that leads to locomotion. We also describe how additional particles can be added to the simple swimmer in situ to assemble multibody swimmers. For multibody swimmers, the stochastic thermal forces may modify the swimming strokes by fragmenting the arm assembled from the smaller particles. Depending on the type of arm fragmentation, the modified strokes can result in significantly faster or slower swimming speed. 


\section{Materials and Methods}

\section{Sample Preparation}

The superparamagnetic particles Dynabeads M-270 and Dynabeads MyOne (Invitrogen) are used. The former particles have characteristic values of $d_{1}=2.8 \pm 0.08 \mu m$ and $\chi_{1}=0.96$, whereas the latter have values of $d_{2}=1.04 \pm 0.02 \mu \mathrm{m}$ and $\chi_{2}=1.4$, as reported by the manufacturer and in literature ${ }^{20,21}$. Both particles have $\psi_{0}=-50 m V^{22}$ and are confirmed to be superparamagnetic $^{21}$. The particles are suspended in $0.1 \mathrm{mM} \mathrm{NaCl}$ solution. The suspension is confined between two coverslips, which have been pretreated with ethyl alcohol and cleaned in plasma cleaner (Harrick Plasma PDC-32G). The particles will settle and remain near the bottom coverslip, where their diffusion and locomotion are hindered ${ }^{23-25}$. Additionally, since both the particles and coverslips are negatively charged, the counterbalance between the electrostatic force and gravitational force confine the particles to a 2-D plane near the bottom of the chamber. The fluctuation of the swimmers in vertical axis is no more than $5 \%$ of the size of the torso particle. The environment is therefore homogeneous in the horizontal plane within which the strokes are confined.

\section{Eccentric Rotating Magnetic (ERM) Field}

The ERM field is generated by an orthogonal set of air-core solenoids. It is an AC magnetic field with a DC field offset: $H_{0}=\left(H_{c}[\cos (2 \pi f t)+\lambda \cos \beta], H_{c}[\sin (2 \pi f t)+\lambda \sin \beta]\right)$, where $H_{c}$ is the magnetic field strength from the circular AC field, $\lambda$ is the DC offset ratio, $\beta$ is the angle of the DC offset, $f$ is frequency and $t$ is time. For the experiments described, the ERM parameters are $H_{c}=270 e, \lambda=\sqrt{2}$, and $\beta=0$ (Agilent N6705). The relationship between swimming stroke and offset ratio is discussed in details in ESI. 


\section{Experimental Setup}

The swimmers are tracked using an optical system that consists of a $100 \times / 1.4$ oil objective (Olympus) and a CCD camera (QImaging). We choose 60 seconds as the tracking time for all swimmers so that the contribution of propulsion to locomotion is much greater (over 10 times) than that of random diffusion for all swimmers. We use 20 replicates for the experimental realization of each type of swimmer.

\section{Diffusion Coefficient}

We use Eqn. (8) to calculate the MSD for all Brownian dynamics (BD) simulations. For the diffusion coefficient used in the equation, only the torso particles are considered. In fact, the diffusion coefficient of a simple two-body swimmer in the horizontal plane only deviates that of a single torso particle by $5 \%$. Considering the contribution of propulsion to locomotion is over 10 times greater than that of the random diffusion for a 60 -second time frame, the added arm particle does not make a noticeable difference when calculating the MSD. Nevertheless, the diffusion coefficient of two torso particles is $5.13 \cdot 10^{-14} \mathrm{~m}^{2} / \mathrm{s}$ and deviates from that of a single torso particle by $28 \%$. The diffusion coefficients of two torso particles are calculated using

the software HydroSub ${ }^{26}$. In the software, the two particles are placed with the average spacing obtained from the BD simulation.

\section{Numerical Schemes}

The numerical scheme for the BD simulation without thermal forces is given by ${ }^{27}$ :

$$
\mathbf{r}_{i}(t+\Delta t)=\mathbf{r}_{i}(t)+\sum_{j} \frac{\mathbf{D}_{i j}\left(\mathbf{r}_{i}(t)\right) \cdot \mathbf{F}_{j}\left(\mathbf{r}_{i}(t)\right)}{k_{B} T} \Delta t
$$

where $\mathbf{r}_{i}(t)$ is the position of particle $i$ at time $t, \mathbf{F}_{j}$ is the force on particle $j, \mathbf{D}_{i j}$ is the diffusion tension taking into account the hydrodynamic interaction mediated by the surrounding fluid, $k_{B}$ is the Boltzmann constant and $T$ is the current experimental absolute temperature. 
The Rotne-Prager tensor ${ }^{28}$ is used for $\mathbf{D}_{i j}$. Similarly the numerical scheme for the BD simulation is given by ${ }^{27}$

$$
\mathbf{r}_{i}(t+\Delta t)=\mathbf{r}_{i}(t)+\sum_{j} \frac{\mathbf{D}_{i j}\left(\mathbf{r}_{i}(t)\right) \cdot \mathbf{F}_{j}\left(\mathbf{r}_{i}(t)\right)}{k_{B} T} \Delta t+\xi_{i}(\Delta t)
$$

where $\xi_{i}(\Delta t)$ is a random force that can be generated at each time step from a Gaussian distribution with zero mean and variance $\left\langle\xi_{i}(\Delta t) \xi_{j}(\Delta t)\right\rangle=2 D_{i j} \Delta t$.

The force on each particle is composed of the magnetic interactions described by micro-mutual-dipolar model (MMDM) ${ }^{29}$ :

$$
\mathbf{F}_{i}=-\mathbf{F}_{j}=\frac{3 \mu_{0}}{4 \pi r^{5}}\left[\left(\mathbf{m}_{1} \cdot \mathbf{r}\right) \mathbf{m}_{2}+\left(\mathbf{m}_{2} \cdot \mathbf{r}\right) \mathbf{m}_{1}+\left(\mathbf{m}_{1} \cdot \mathbf{m}_{2}\right) \mathbf{r}-\frac{5\left(\mathbf{m}_{1} \cdot \mathbf{r}\right)\left(\mathbf{m}_{2} \cdot \mathbf{r}\right)}{r^{2}} \mathbf{r}\right]
$$

Here $\mathbf{r}$ is the vector from particle $i$ to $j$ and $r$ is the norm of said vector, $\mu_{0}$ is vacuum permeability and $\mathbf{m}_{i}$ is the dipole moment on particle $i$ that satisfies

$$
\begin{array}{r}
\mathbf{m}_{i}=\frac{4}{3} \pi a_{i}^{3} \chi_{i}\left(\mathbf{H}_{0}+\sum_{k=1, k \neq i}^{N} \mathbf{H}_{d i p}\left(\mathbf{R}_{i}-\mathbf{R}_{k}\right)\right) \\
\mathbf{R}_{i}=\frac{\int_{V} d V\left(\mathbf{r}_{i}+\mathbf{q}\right)\left|\mathbf{H}_{0}+\sum_{k=1, k \neq i}^{N} \mathbf{H}_{d i p}\left(\mathbf{r}_{i}+\mathbf{q}-\mathbf{r}_{k}\right)\right|^{3}}{\int_{V} d V\left|\mathbf{H}_{0}+\sum_{k=1, k \neq i}^{N} \mathbf{H}_{d i p}\left(\mathbf{r}_{i}+\mathbf{q}-\mathbf{r}_{k}\right)\right|^{3}}
\end{array}
$$

simultaneously. In the above equations, $\mathbf{H}_{d i p}(\mathbf{r})=\frac{1}{4 \pi}\left(\frac{3 \mathbf{r}(\mathbf{m} \cdot \mathbf{r})}{r^{5}}-\frac{\mathbf{m}}{r^{3}}\right)$ is the dipole-induced magnetic field,,$N$ is the number of spheres, $\mathbf{r}_{i}$ is the position of the dipole moment for particle 
$i, \mathbf{H}_{\mathbf{0}}$ is the applied magnetic field, $\chi_{i}$ is the susceptibility of the particle $i, \mathbf{R}_{n}$ are the positions of the dipole moments, $\mathbf{q}$ is the position of the current integral volume $\mathrm{d} V$, and the integral goes over the entire volume of particle $i$. We adopt Derjaguin-Landau-Verwey-Overbeek (DLVO) theory ${ }^{30}$ to describe the electrostatic repulsion:

$$
\mathbf{F}_{1}=-\mathbf{F}_{2}=\left(32 \pi k T d_{h} \rho_{\infty} \gamma^{2} / \kappa^{2}\right) e^{-\kappa\left(r-d_{a}\right)}
$$

where $d_{h}=\frac{2 d_{1} d_{2}}{d_{1}+d_{2}}$ is the harmonic mean of the particle diameters, $d_{a}=\frac{d_{1}+d_{2}}{2}$ is the arithmetic mean of the particle diameters, $\rho_{\infty}$ is the number density of ions in the bulk solution, $\kappa$ is the reciprocal of the Debye length, and $\gamma=\tanh \left(z e \psi_{0} / 4 k_{B} T\right)$ is the reduced potential, where $\psi_{0}$ is the surface potential and $e$ is the unit charge. Both particles have the same surface potential and are thus not differentiated here. We neglect the van der Waals term in DLVO theory because its contribution is negligible for the situation in which the particle surface separation is sufficiently large ${ }^{20}$. We use 20 replicates for the simulation of each type of swimmer.

\section{Magnetic Field Strength Distribution}

The magnetic field strength distribution is calculated by solving Laplace's equation for magnetostatics using a smoothed representation of the magnetic susceptibility ${ }^{31}$. The gradient of the magnetic field correlates with the magnetic force density which is calculated using Maxwell stress tensor.

$$
\mathbf{f}_{m}=\nabla \cdot \boldsymbol{\sigma}_{m}=\nabla \cdot\left[\mu_{0}\left(\mathbf{H H}-\frac{1}{2} H^{2} \mathbf{I}\right)\right]
$$




\section{Results and Discussions}

\section{The Simple Swimmer and Non-reciprocal Motion}

To experimentally realize this swimmer, we utilize two paramagnetic colloidal particles of sizes of $2.8 \mu \mathrm{m}$ and $1.04 \mu \mathrm{m}$. The dynamics can be numerically simulated by an equation of motion that includes magnetic, hydrodynamic, and electrostatic forces ${ }^{20}$. It is known that a pair of paramagnetic particles placed in a classic rotating magnetic (CRM) field will acquire an attractive magnetic interaction and torque, causing the pair to rotate in place with the external magnetic field ${ }^{20}$. We apply an eccentric rotating magnetic (ERM) field: $H_{0}=\left(H_{c}[\cos (2 \pi f t)+\lambda \cos \beta], H_{c}[\sin (2 \pi f t)+\lambda \sin \beta]\right)$, by adding a DC offset ratio $\lambda=\frac{|O M|}{H_{c}}$

to a CRM field (Fig. 1B and C, movie S1). Here $H_{c}$ is the magnetic field strength of the CRM

field and $|O M|$ is the DC offset, $\beta$ is the angle of the DC offset, $f$ is frequency and $t$ is time. Under an ERM field with a large eccentric ratio, the smaller particle will be directed to orbit on one side of the larger particle with a semicircular trajectory that encloses a nonzero area (Fig. 1D). As an analogy to macroscale swimming, we define the smaller particle as the "arm" particle due to its larger sweep angle which drives the larger "torso" particle. During the recovery phase, the arm first sweeps upward in close proximity to the torso (Point $\mathrm{P}$ to $\mathrm{Q}$ ). During the stroke phase, the low magnetic field strength prevents the arm from following the route in the previous phase (Point $\mathrm{P}$ to $\mathrm{Q}$ via $\mathrm{M}$ ). For high frequencies $(f>1 \mathrm{~Hz})$, phase lag emerges between the arm and the external field during the stoke phase. The attraction between the two particles can no longer hold them together, and therefore, they begin to repel each other at approximately the largest angle the arm can reach (Point $\mathrm{Q}$ to $\mathrm{M}$ ). The rotating field eventually catches up with the arm particle, causing the arm to move downward and finish the stroke (Point $\mathrm{M}$ to $\mathrm{P}$ ). Due to the nonzero enclosed area, the asymmetric hydrodynamic interaction between the torso and the arm generates net propulsion, driving them to translate together as a collective pair (Fig. 1E). The magnetic torque functions as a "hinge" to control the sweep angle $(\Delta \phi)$, defined as the maximum angle within which the arm particle is able to orbit near the torso particle, and the radial magnetic force functions as a "stretchable rod" to control the magnitude 
of the sweep radial distance $(\Delta \mathrm{r})$, defined as the difference between the maximum and minimum distances between the arm and torso centers of mass (Fig. 1D). Since the strokes are confined in 2-D (See Materials and Methods), $\Delta \mathrm{r}$ and $\Delta \phi$ are the only two degrees of freedom involved in the arm motion. The frequency modifies the arm trajectory in two ways. First, the sweep angle decreases with increasing frequency due to increased fluid drag on the arm particle. Second, the sweep radial distance initially increases and then decreases with increasing frequency (Fig. 1F).

A

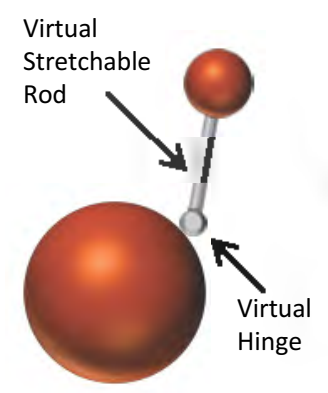

D

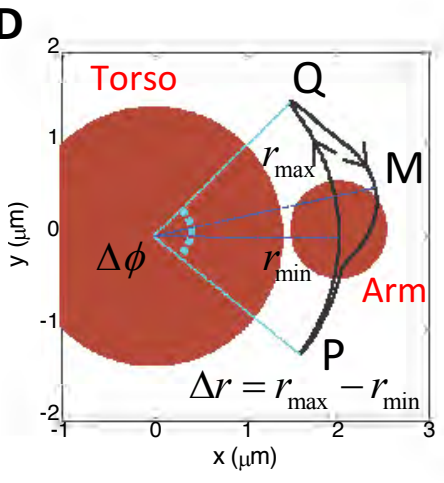

B

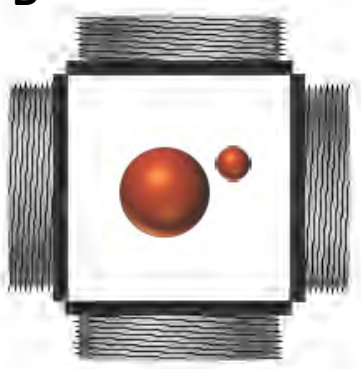

$\mathbf{E}$

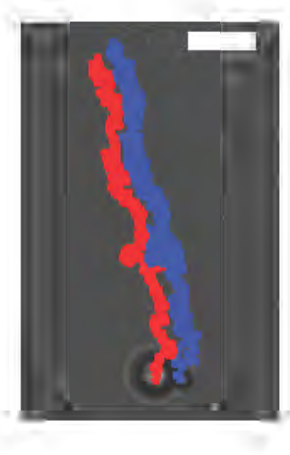

C

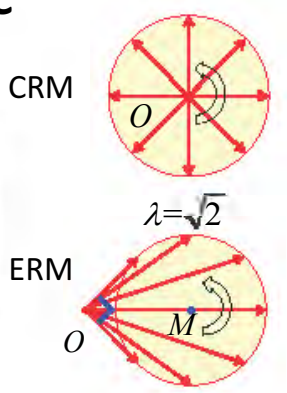

$\mathbf{F}$

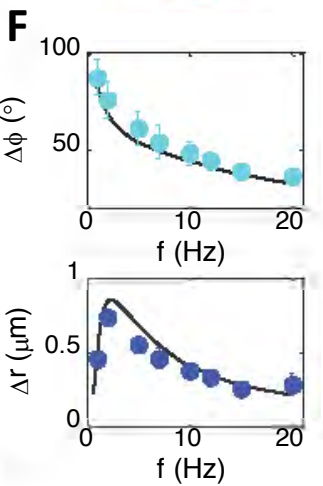

Fig. 1. The two-body swimmer under an ERM field. (A) Schematics of the two-body swimmer. (B) Experimental realization of the swimmer using an ERM field. (C) The difference between a CRM field and an ERM field with $\lambda=\sqrt{2}$. The red arrows correspond to the magnetic field vectors at different time steps. (D) The trajectory of the arm particle in the frame of the torso particle obtained from simulation. The arm orbits clockwise. (E) The trajectory of both particles in 60 seconds. Scale bars represents $5 \mu \mathrm{m}$. (F) The sweep angle (top) and sweep radial distance (bottom) of the smaller arm particle under different frequencies. Dots with error bars (cyan and blue) correspond to experiment results, and the solid lines (black) correspond to simulation results. The ERM field used in (D) $\sim(\mathbf{F})$ is $H_{c}=27 O e$ and $f=10 \mathrm{~Hz}$. 
The arm trajectory directly correlates with the displacement per cycle (DPC), $\delta / f t$, which can be directly obtained from a Brownian dynamics (BD) simulation without thermal forces (Fig. 2A). The largest DPC is observed approximately when the arm trajectory encloses the largest area. The non-monotonic behavior of $\delta / f t$ stems from the non-monotonic behavior of the sweep radial distance. The mean square displacement (MSD) for the swimmer without orientation loss is given by ${ }^{9}$

$$
\left\langle\delta^{2}\right\rangle=4 D t+U^{2} t^{2}
$$

with contributions from both propulsion and stochastic forces, where $U$ stands for the propulsion speed without consideration of thermal forces (DPC multiplied by $f$ in Fig. 2A). The diffusion coefficient considered is that of the torso particle $D_{1}=\frac{k_{B} T}{3 \pi \eta d_{1}}$ (See Materials and Methods), where $\eta$ is the apparent viscosity of the surrounding fluid, $k_{B}$ is Boltzmann's constant and $T$ is given in absolute temperature. The square roots of the MSD (RMSD) values obtained from Eqn. (8) and experimental results are in very good agreement (Fig. 2B).

The locomotion direction of this simple swimmer is determined by the direction of the DC offset of the field, OM (Fig. 1C). The stochastic force does not noticeably affect the orientation of motion $\theta$ (Fig. 2B inset), which confirms that the swimmer is free of orientation loss. The MSD over time distinguishes ballistic locomotion of a swimmer from simple stochastic diffusion of a single torso particle (Fig. 2C). Good agreement is observed between the simulation and experimental results for both the swimmer and the single particle. Unlike chemotactic swimmers that experience a transition from ballistic locomotion to random walk at longer times due to orientation loss ${ }^{9}$, this mechanical swimmer is directed by the magnetic field and always maintains ballistic motion when the field is on. 

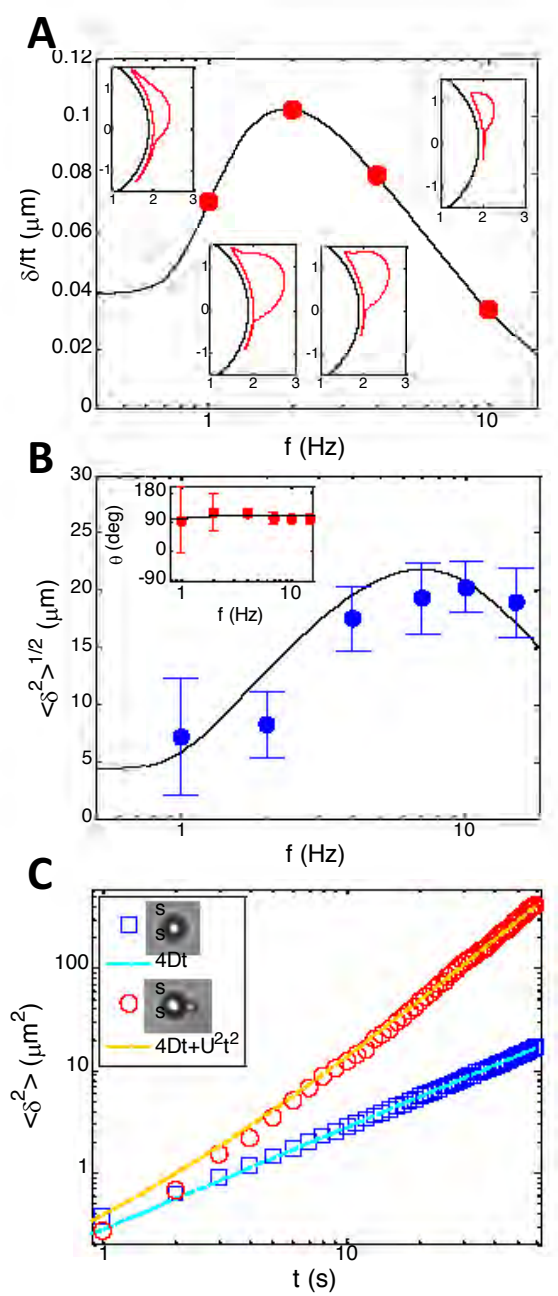

Fig. 2. Swimmer locomotion at different frequencies. (A) DPC at different frequencies under $H_{c}=270 e$ obtained from simulation. Insets show arm trajectories in the torso frame for the four markers in corresponding left-to-right order. (B) RMSD of the swimmer at 60 seconds under different frequencies. The circular markers with error bars (blue) represent experimental results, and the solid line (black) represents predicted values obtained from Eqn. (8). The inset at the top shows the mean angle of locomotion at 60 seconds. The square markers with error bars (red) represent experimental results, and the solid line represents predicted values. (C) MSD of a single particle and a swimmer over 60 seconds. 


\section{Multibody Swimmers and the Effect of Thermal Forces}

We further studied more complicated microswimmers with multiple torsos and arms, which can be assembled easily from simpler members of the family (movie S2). Here, " $n_{1} t n_{2} a$ " is used to represent a microswimmer with $n_{1}$ torso particles and $n_{2}$ arm particles. For multibody swimmers, BD simulations including thermal forces result in very good agreement with the experimental measurements of RMSD (Fig. 3A). The difference between the BD simulations with and without thermal forces is the fragmentation of swimmer arm, which is defined as the case where an inter-particle distance between nearest neighbors is larger than the diameter of the arm particle (Fig. 3A, movie S3 and S4). In particular, arm fragmentation decreases the swimming speed of $1 \mathrm{t} 2 \mathrm{a}$ by $24 \%$, increases that of $1 \mathrm{t} 4 \mathrm{a}$ by $30 \%$, and increases that of $2 \mathrm{t} 4 \mathrm{a}$ by $125 \%$. With the arm fragmentation to enhance the swimming speed, the $2 \mathrm{t} 4 \mathrm{a}$ swimmer swims at $0.84 \mu \mathrm{m} / \mathrm{s}$ while the $1 \mathrm{t} 2 \mathrm{a}$ swimmer swims at $0.34 \mu \mathrm{m} / \mathrm{s}$. Similarly, RMSDs were calculated using Eqn. (8) for BD simulation results, where the diffusion coefficient of two torso particles was considered (See Materials and Methods).

Arm fragmentation changes the swimming strokes. When the arm reaches the maximum sweep angle, the ERM field approaches the perigee, where the magnetic field strength holding the particles together is the weakest. The end particles in the arm are easily segregated from the swimmer and remain separated while the other arm particles make a downward stroke. As a result, the sweep angle of end particles in the arm are not commensurate with those of the other arm particles in sweep angle (Fig. 3B and D).

The weak arm affinity is directly caused by the multipolar magnetic induction inside the swimmer (Fig. 3C and E). The magnetic field gradient between the torso and the two adjacent arm particles 1 and 2 is much stronger than that between arm particles 3 and 4, and there is negligible magnetic field gradient between arm particles 1 and 2. An additional torso particle further weakens the affinity among all of the arm particles, and increase the probabilities of existing in each fragmentation type respectively.

We define two types of fragmentation: Type 1, where the furthest arm particle is released and Type 2, where the torso is separated from the arm particles (Fig. 4A and C). Type 1, Type 2, 
and simultaneous Type 1 and 2 fragmentations occur with different fractional occurrences for different swimmers (Fig. 3A). Arm fragmentation does not always occur for each cycle. The standard deviations of all the fractional occurrences shown in Fig. 3A are no more than 5\% with 20 replicates for each swimmer, indicating that the fractional occurrences almost remain constant for each swimmer.
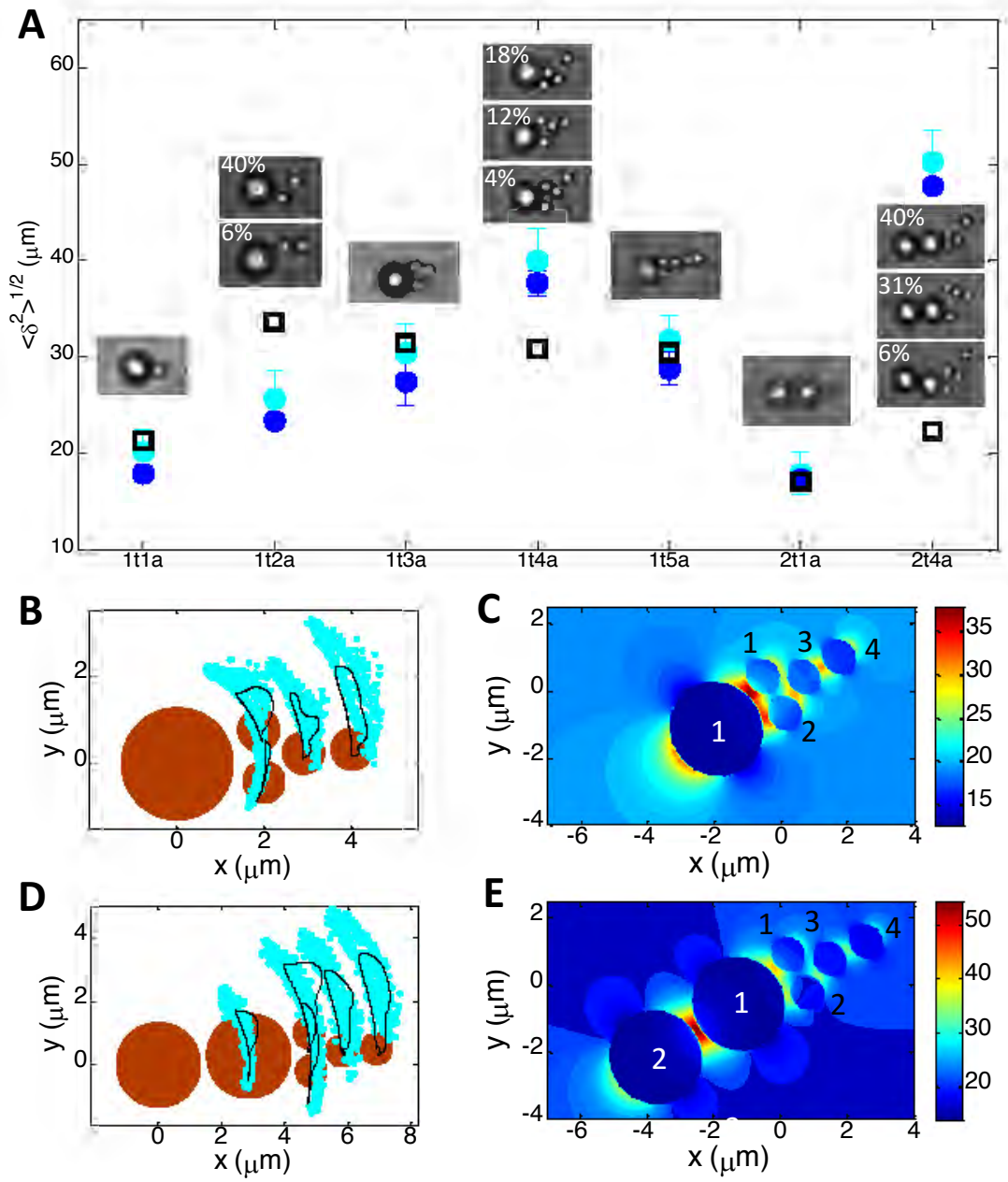

Fig. 3. Swimmers with multiple torsos and arms. (A) RMSD of swimmers with different numbers of torsos and arms at 60 seconds. The square markers (black) represent BD simulation results without thermal forces, the filled circles (blue) with error bars represent BD simulation results with thermal forces, and filled circles (cyan) with error bars represent experimental results.

Snapshots of initial configurations for different swimmers are inserted correspondingly. Snapshots of the $1 \mathrm{t} 2 \mathrm{a}, 1 \mathrm{t} 4 \mathrm{a}$ and $2 \mathrm{t} 4 \mathrm{a}$ swimmers under fragmentation are shown along with the fractional occurrences of each fragmented type. (B) Arm trajectory in the torso frame for 1t4a. (C) 
Magnetic field strength distribution in the $x-y$ plane for 1 t4a when the swimmer's long axis reaches the largest angle. (D) Arm trajectory in the frame of the torso at the end for $2 \mathrm{t} 4 \mathrm{a}$. (E)

Magnetic field strength distribution in the $x-y$ plane for $2 t 4 a$ when the swimmer's long axis reaches the largest angle. For (B) and (D), solid curves correspond to BD simulation results without thermal forces and filled circles (cyan) experimental results. For (C) and (E), the color bars represent the magnetic field strength in units of Oe. The ERM field used is $H_{c}=27 O e$ and

$$
f=10 H z \text {. }
$$

\section{Arm Fragmentation}

It was observed that arm fragmentation enhances the swimming speed for $1 \mathrm{t} 4 \mathrm{a}$ and $2 \mathrm{t} 4 \mathrm{a}$ but decreases that of 1t2a. The arm fragmentation can be characterized by arm bending angle $\alpha$, which for one torso swimmer is defined by the angle formed by the torso particle and two arm particles at the far right (Figs. 4A and $\mathrm{C}$ insets). We used simulations to investigate the statistics of arm bending angles for $1 \mathrm{t} 2 \mathrm{a}$ and $1 \mathrm{t} 4 \mathrm{a}$ swimmers. For these swimmers, Type 1 fragmentation features a large positive $\alpha$, Type 2 fragmentation a large negative $\alpha$ and simultaneous Type 1 and 2 fragmentation features a small positive $\alpha$ (Figs. 4A and C). For swimmers with longer arms, more complex arm fragmentation types may also occur.

Generally either Type 1 or Type 2 fragmentation generates smaller propulsion than no fragmentation, whereas simultaneous Type 1 and 2 fragmentation generates a markedly larger propulsion (Figs. 4B and D). The simultaneous Type 1 and 2 fragmentation is not feasible for $1 \mathrm{t} 2 \mathrm{a}$, thus its swimming speed is decreased by the modified strokes caused by arm fragmentation. This also explains why the speed enhancement to $2 \mathrm{t} 4 \mathrm{a}$ is more significant than that to $1 \mathrm{t} 4 \mathrm{a}$. The fractional occurrences for each of the arm fragmentation type obtained from simulation also show good agreement with those obtained from experiment (Figs. 4B and D, Fig 3A). The thermal forces are stochastic, but the fractional occurrences for each type of the arm fragmentation resulted from the balance between magnetic forces and thermal forces are determinant for a swimmer. The thermal forces modify the swimming strokes via arm fragmentation, leading to enhancement or reduction of swimming speed depending on the fractional occurrences for each fragmentation type. 

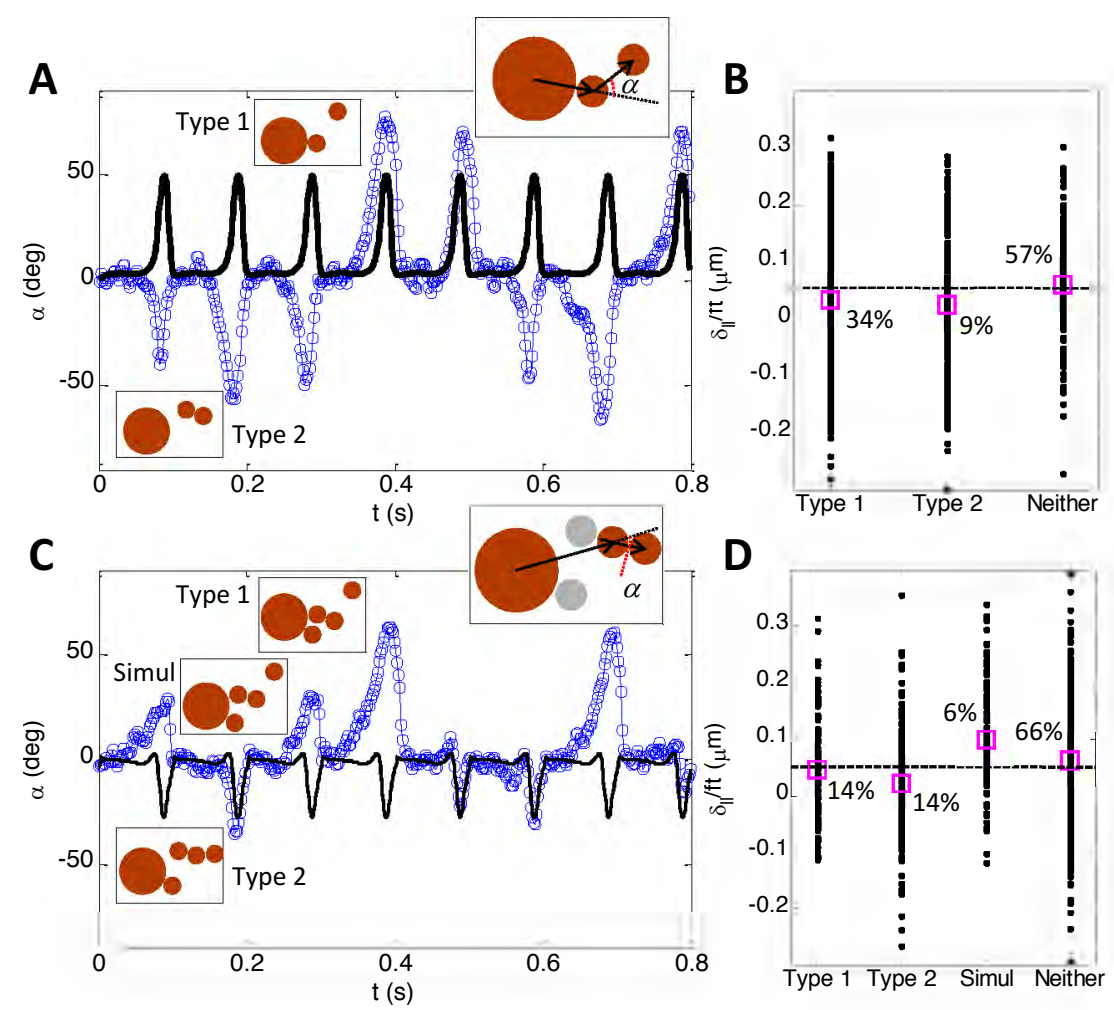

Fig. 4. Analysis of different types of fragmentation using arm bending angle. (A) The arm bending angle for a 1t2a swimmer within 8 cycles. (B) The statistics of DPC for different types of arm fragmentation for a 1t2a swimmer. (C) The arm bending angle for a $1 \mathrm{t} 4 \mathrm{a}$ swimmer within 8 cycles. (D) The statistics of DPC for different types of arm fragmentation for a 1t4a swimmer. For (A) and (C), the solid curves (black) represent simulation results without stochastic forces, and the circles (blue) simulation results with stochastic forces. The insets are schematics of arm bending angle for each swimmer. Snapshots of different types of fragmentation are superimposed for guidance. For (B) and (D), the dashed lines (black) correspond to simulation results without stochastic forces, the filled circles (black) and squares (magenta) simulation results with stochastic forces and their arithmetic means respectively, and the percentages the probabilities of existing in each fragmented type. The ERM field used is $H_{c}=27 O e$ and

$$
f=10 H z \text {. }
$$




\section{Conclusions}

We have demonstrated the design of a simple mechanical microswimmer that consists of two paramagnetic particles of different sizes. The feasibility of the swimmer is experimentally confirmed using an ERM field with strong anisotropy to break the time reversibility. Designed for homogeneous Newtonian fluids, this mechanical swimmer consists of the smallest number of rigid components as no complete swimmer will form when the number of rigid components is further decreased. This simple swimmer adopts the motion of breaststroke, where the sweep of an arm near one side of a torso is used to generate propulsion. The strokes are constrained within a horizontal plane where the property of the surrounding fluid is homogeneous. This significantly differs from the swimming motion achieved using the heterogeneity of the fluid near a surface ${ }^{15}$,

${ }^{16}$, which is analogous to the motion of freestyle or butterfly stroke. The fact that the swimming motion presented here is not dependent on the surface is confirmed by the simulation.

From this swimmer we have introduced a family of swimmers with multiple components, cooperatively connected together by magnetic forces. Due to multipolar magnetic induction, the magnetic attractive forces among the arm particles become weak and comparable to thermal forces. The multibody swimmers fragment their arms in different configurations as a result of the thermal forces. The arm fragmentation affects the swimming strokes and therefore the swimming speed.

When considering the balance between viscous force and inertial force, the Scallop theorem shows that swimming is difficult to achieve at low Re numbers because of the requirement of non-reciprocal body motion ${ }^{2}$. When thermal forces are involved, reciprocal motion becomes useful since reciprocal actuators experience enhanced diffusion in a fluctuating environment ${ }^{32}$. Therefore thermal forces enhance the locomotion of reciprocal swimmers. Here the modified strokes of the multibody swimmers indicate that thermal forces also affect the locomotion of non-reciprocal swimmers. The effect can be enhancement or reduction, depending on the probabilities of different arm fragmentation types. Such observations provide insight into the role of stochastic forces in locomotion at low Reynolds number. 


\section{Acknowledgements}

The authors thank Dichuan Li, Julie Byrom, Hojin Jeon and Matteo Pasquali for insightful discussions. This work was supported by the National Science Foundation under Grant No. CBET-1705703.

\section{References}

1. R. Dreyfus, J. Baudry, M. L. Roper, M. Fermigier, H. A. Stone and J. Bibette, Nature, 2005, 437, 862-865.

2. E. M. Purcell, Am. J. Phys., 1977, 45, 3-11.

3. N. Vandenberghe, J. Zhang and S. Childress, J. Fluid Mech., 2004, 506, 147-155.

4. T. M. Williams, R. W. Davis, L. A. Fuiman, J. Francis, B. J. Le, Boeuf, M. Horning, J. Calambokidis and D. A. Croll, Science, 2000, 288, 133-136.

5. S. C. Schuster and S. Khan, Annual review of biophysics and biomolecular structure Annu. Rev. Biophys. Biomol. Struct., 1994, 23, 509-539.

6. J. Elgeti, U. B. Kaupp and G. Gompper, Biophys. J., 2010, 99, 1018-1026.

7. I. Gibbons, J. Cell Biol., 1981, 91, 107s-124s.

8. T. Qiu, T.-C. Lee, A. G. Mark, K. I. Morozov, R. Münster, O. Mierka, S. Turek, A. M. Leshansky and P. Fischer, Nat. Commun., 2014, 5.

9. J. R. Howse, R. A. L. Jones, A. J. Ryan, T. Gough, R. Vafabakhsh and R. Golestanian, Phys. Rev. Lett., 2007, 99, 048102.

10. S. Palagi, A. G. Mark, S. Y. Reigh, K. Melde, T. Qiu, H. Zeng, C. Parmeggiani, D. Martella, A. Sanchez-Castillo, N. Kapernaum, F. Giesselmann, D. S. Wiersma, E. Lauga and P. Fischer, Nat. Mater., 2016, 15, 647-653.

11. B. J. Williams, S. V. Anand, J. Rajagopalan and M. T. A. Saif, Nat. Commun., 2014, 5.

12. A. Najafi and R. Golestanian, Phys. Rev. E, 2004, 69, 4.

13. M. Leoni, J. Kotar, B. Bassetti, P. Cicuta and M. C. Lagomarsino, Soft Matter, 2009, 5, 472-476.

14. B. U. Felderhof, Phys. Fluids, 2017, 29, 011901. 
15. C. E. Sing, L. Schmid, M. F. Schneider, T. Franke and A. Alexander-Katz, Proc. Nat. Acad. Sci., 2010, 107, 535-540.

16. P. Tierno, R. Golestanian, I. Pagonabarraga and F. Sagues, Phys. Rev. Lett., 2008, 101, 218304.

17. P. Tierno, O. Guell, F. Sagues, R. Golestanian and I. Pagonabarraga, Phys. Rev. E, 2010, 81,9 .

18. P. J. Vach and D. Faivre, Sci.Rep., 2015, 5, 9364.

19. F. Z. Temel and S. Yesilyurt, Magnetically actuated micro swimming of bio-inspired robots in mini channels, IEEE Int. Conf. Mechatronics., 2011.

20. D. Du, D. Li, M. Thakur and S. Biswal, Soft Matter, 2013, 9, 6867-6875.

21. G. Fonnum, C. Johansson, A. Molteberg, S. Morup and E. Aksnes, J. Magn. Magn. Mater., 2005, 293, 41-47.

22. D. C. Li, C. N. Lam and S. L. Biswal, Soft Matter, 2010, 6, 239-242.

23. M. A. Bevan and D. C. Prieve, J. Chem. Phys., 2000, 113, 1228-1236.

24. V. Michailidou, G. Petekidis, J. Swan and J. Brady, Phys. Rev. Lett., 2009, 102, 068302.

25. J. W. Swan and J. F. Brady, Phys. Fluids, 2007, 19, 113306.

26. J. Garcia de la Torre and B. Carrasco, Biopolymers, 2002, 63, 163-167.

27. D. L. Ermak and J. A. McCammon, J. Chem. Phys., 1978, 69, 1352-1360.

28. J. Rotne and S. Prager, J. Chem. Phys., 1969, 50, 4831-4837.

29. D. Du and S. L. Biswal, Phys. Rev. E, 2014, 90, 033310.

30. J. Israelachvili, Intermolecular and Surface Forces, Academic Press, San Diego, CA, 1992.

31. D. Du, F. Toffoletto and S. L. Biswal, Phys. Rev. E, 2014, 89, 043306.

32. E. Lauga, Phys. Rev. Lett., 2011, 106, 4. 